\title{
Using Narrowing to Test Maude Specifications ${ }^{\star}$
}

\author{
Adrián Riesco \\ Facultad de Informática, Universidad Complutense de Madrid, Spain \\ ariesco@fdi.ucm.es
}

\begin{abstract}
Testing is one of the most important and most time-consuming tasks in the software developing process and thus techniques and systems to automatically generate and check test cases have become crucial. In previous work we have presented techniques to test membership equational logic specifications; these techniques consist of two steps: first several ground terms are generated by using all the available constructor symbols in a breadth-first search, and then these terms are processed to check whether they fulfill some properties. This approach presents the drawback of separating two related processes, thus examining several terms that are indistinguishable from the point of view of testing. We present here a narrowing-based test-case generator that improves the performance of the tool and extends its use to rewriting logic specifications. First, we present two mechanisms to improve the narrowing commands currently available in Maude to use conditional statements and equational modules. Then, we show how to use these new narrowing commands to perform three different approaches to testing for any Maude specification: code coverage, property-based testing, and conformance testing. Finally, we present trusting mechanisms to improve the performance of the tool. We illustrate the tool by means of an example.
\end{abstract}

Keywords: testing, Maude, narrowing, coverage, property, conformance.

\section{Introduction}

Testing is a technique for checking the correctness of programs by means of executing several inputs and studying the obtained results. Testing is one of the most important stages of the software-development process, but it also is a very time-consuming and tedious task, and for this reason several efforts have been devoted to automate it [2]1. Basically, we can distinguish two different approaches to testing: glass-box testing [13|24, that uses the specific statements of the system to generate the most appropriate test cases, and black-box testing 3214/5], that considers the system as a black box with an unknown structure and where a specification of the system is used to generate the test cases and check their correctness. We can also distinguish different kinds of testing depending on how the test cases are obtained: they can either be ground terms

* Research supported by MEC Spanish project DESAFIOS10 (TIN2009-14599-C03-

01) and Comunidad de Madrid program PROMETIDOS (S2009/TIC1465). 
that are later executed to check the obtained results or terms with variables that are symbolically executed [20] to find the most appropriate values to test the program. While the former generates in general more test cases (because it just combines constructors to build terms) they can be illegal (input that can never be used in real executions) and equivalent (different test cases check the same statements), the latter generates less but more accurate test cases.

Maude [8] is a high-level language and high-performance system supporting both equational and rewriting logic computation for a wide range of applications. Maude modules correspond to specifications in rewriting logic 22. This logic is an extension of equational logic; in particular, Maude functional modules correspond to specifications in membership equational logic $[3]$, which, in addition to equations, allows the statement of membership axioms characterizing the elements of a sort. Rewriting logic extends membership equational logic by adding rewrite rules, that represent transitions in a concurrent system. Maude system modules are used to define specifications in this logic. The current version of Maude supports a limited version of narrowing [31, a generalization of term rewriting that allows to execute terms with variables by replacing pattern matching by unification, for some unconditional rewriting logic theories without memberships. This limitation is dropped in this work by using a program transformation and by checking separately the conditions.

As part of an ongoing project to test and debug Maude specifications, we have implemented a declarative debugger for Maude specifications [28, that allows the user to debug both wrong (incorrect results obtained from a valid input) and missing (incomplete results obtained from a valid input) answers in any Maude specification, and a test case generator for functional modules 27. The testing approach used in that paper consists of different phases: first, the module is preprocessed to obtain the statements used by the functions being tested; then, terms are generated by using a breadth-first search that takes into account the constructor information provided by the user, and then each of these terms is executed step-by-step to check the used statements. However, this approach uses ground terms and, as explained above, presents an important drawback: since the test cases are not generated following the structure of the program but just the available constructors, most of them apply the same statements, hence consuming most of the time and preventing more complex terms from being checked due to the time and space constraints. This problem is solved here by symbolically executing terms with variables with narrowing.

We present in this paper a program transformation to test Maude functional modules by using narrowing, a strategy to use membership axioms and conditional statements in the narrowing process 1 and the adaptation of three testing techniques to Maude: two white-box approaches (one selects a set of test cases whose correctness must be checked by the user, while the other one checks

\footnotetext{
${ }^{1}$ This strategy allows all kinds of conditions: rewrite and equational conditions, solved by narrowing (the latter, which includes equational and matching conditions, requires a previous transformation), and membership conditions, solved by using unification.
} 
whether a property holds in the specification) and one black-box mechanism (conformance testing). In the first case, in addition to other criteria described in 27, we have adapted a new criterion to select the set of test cases to be checked by the user in system modules, which is based on modified condition decision coverage [19] and checks the negative information (the rules that are not applied). Finally, we enhance the performance of the tool by providing trusting techniques that prevent the system from taking into account some statements. The transformation, the extension of the narrowing process, and the testing strategies have been implemented in a Maude prototype by using its meta-level capabilities, that allow to manipulate Maude modules and statements as usual data. Moreover, it also provides support for some predefined modules and attributes, such as owise, that indicates that the current equation is only used when the rest of equations cannot be applied.

The rest of the paper is organized as follows: Section 2 presents some related work and its relation with our system. Section 3 introduces Maude and narrowing, Section 4 describes a module transformation that allows us to use narrowing on Maude functional modules, while Section 5 presents how to use conditional rules in the narrowing process. Section [6 illustrates how the techniques described in the previous sections are used to generate test cases, while Section 7 presents some trusting techniques to improve the performance of the system. Finally, Section 8 concludes and outlines some future work. The source code of the tool, examples, related papers, and much more information is available at http://maude.sip.ucm.es/testing/.

\section{Related Work}

Different approaches to testing for declarative languages have been proposed in the literature. As explained in the introduction, test cases can be checked in different ways: executing ground test cases or symbolically executing terms with variables.

The first approach is followed by Smallcheck [30, a property-driven Haskell tool that considers that most of the errors can be found by using only a few constructors, and thus it generates all the possible combinations of constructors given a (usually small) bound on the size of the test cases. Another tool following this ground approach is Quickcheck [7, a test-case generator developed for Haskell specifications where the programmer writes assertions about logical properties that a function should fulfill; test cases are randomly generated by using the constructors of the data type (in contrast to the complete search performed by Smallcheck) to test and attempt to falsify these assertions. The project, started in 2000, has been extended to generate test cases for several languages such as Java, C++, Erlang, and several others. Finally, Easycheck [6] is a test-case generator for Curry that takes advantage of the non-determinism of functional-logic programs to generate a tree of potential test cases, that is later traversed to list only the most interesting ones.

The second approach has been applied by Lazy Smallcheck 30. (an improvement of a previous system called SparseCheck), a library for Haskell to test 
partially-defined values that uses a mechanism similar to narrowing to test whether the system fulfills some requirements. Another way of achieving symbolic execution is by considering that the statements in the program under test introduce constraints on the variables, an approach followed by PET 15, that uses Constraint Logic Programming to generate test cases satisfying some coverages on object-oriented languages. Finally, narrowing has been used to verify security protocols [21]18, symbolically exploring the state space trying to find a flow in the protocol.

The previous version of our approach is quite similar to Smallcheck: we generate the complete search space given the constructors and a bound, but we use them for both white-box and black-box testing, while Smallcheck only tries to disprove some properties. Note that, on the one hand, the strategies in our previous system could possibly be improved by following an approach similar to Easycheck, while on the other hand we can consider that the current narrowing approach is another way of pruning the tree of possible terms, making our approach similar to it. Regarding Quickcheck, it is an industrial tool with several heuristics and a lot of experience in testing, and hence it presents a better performance than our tool, that we try to improve by providing trusting mechanisms to the user. On the other hand, an advantage of our tool is the computation of test cases fulfilling different coverage criteria, which allows the user to test the specification by checking test cases "by hand" even when no properties over the specification are stated, and the usage of Maude as both a specification and implementation language, which allows to perform conformance testing using a previously tested Maude module as specification. Moreover, both Quickcheck and our tool implement the shrinking mechanism, that consists of returning the simplest form of a term that detects a bug in the program; in our case it is implemented by performing a breadth-first search using narrowing steps, that will find the simplest term (w.r.t. the number of steps) reproducing the buggy behavior. The more similar approach to ours is Lazy Smallcheck; both are narrowing-based experimental tools that focus on research rather than in efficiency, and thus they present a similar performance; however, Smallcheck is only applied to propertybased testing. PET provides a coverage of the statements in Java-like programs, but it does not allow the user to state properties or check the correctness of the system against a specification. Finally, the verification of security protocols focus on a specific problem and cannot be compared with the rest of tools.

Note that, in general, each system only focus in one testing approach: coverage, properties, or conformance. Maude features allow us to implement a wide range of testing techniques: we can manipulate its modules to perform white-box testing by using its meta-level capabilities; its analysis tools (such as the search command) ease the testing of properties; and Maude programs can be used as specification of others.

\section{Preliminaries}

This section introduces Maude and its narrowing mechanisms 9 . 


\subsection{Maude}

Maude functional modules [8, Chap. 4], with syntax fmod ... endfm, are executable membership equational specifications that allow the definition of sorts (by means of keyword sort(s)); subsort relations between sorts (subsort); operators (op) for building values of these sorts, giving the sorts of their arguments and result, and which may have attributes such as being associative (assoc) or commutative (comm), for example; memberships (mb) asserting that a term has a sort; and equations (eq) identifying terms. Both memberships and equations can be conditional (cmb and ceq). Maude system modules [8, Chap. 6], introduced with syntax mod ... endm, are executable rewrite theories 22. A system module can contain all the declarations of a functional module and, in addition, declarations for rules ( $r l$ ) and conditional rules (crl).

An important characteristic of Maude functional modules is that sorts are grouped into equivalence classes called kinds; that is, all the sorts related by a subsort relation belong to the same kind [8]. Intuitively, terms with a kind but without a sort represent undefined or error elements. We will make extensive use of kinds to indicate that variables may have any sort when performing unification; the proper sorts of the variables will be later checked by means of membership axioms.

We introduce Maude modules with an example; variable declarations are not shown because of space constraints, but assume they are defined at the sort level. We specify ordered lists of natural numbers in the following module:

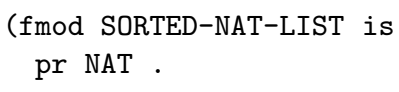

We use the sort NatList, with constructors nil and _._, for generic lists and SortedList for sorted lists, which are a subsort of NatList:

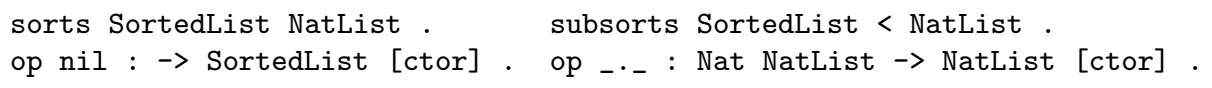

We use membership axioms to characterize nonempty sorted lists. They indicate that the singleton list is ordered (ol1) and that a larger list is ordered if the first element is equal to or smaller than the second one and the rest of the list is also ordered (o12):

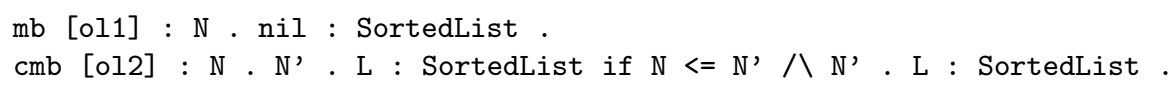

We also specify a function ins-sort that sorts a list by inserting the elements in an ordered fashion by using the auxiliary function ins-list:

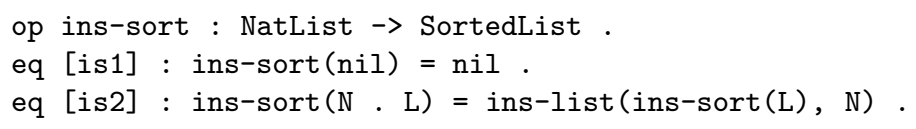

This function returns the singleton list when inserting an element into the empty list, and otherwise it distinguishes whether the first element in the list is smaller or not: 


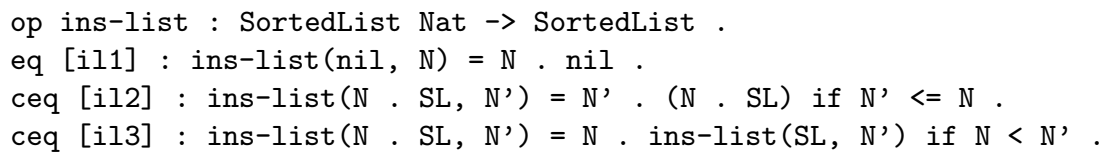

Since we are also interested on testing system modules, we use this module to specify how processes enter into a critical section in the following system module CS. We consider that processes are represented by their priority (the smaller the number the higher the priority), and hence lists of natural numbers stand for lists of processes:

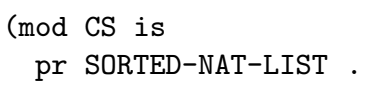

We define the sort NatSoup for an associative and commutative multiset built with the operators mtSoup and _, _; the sort NatWithEmpty for a supersort of the natural numbers with an extra element empty; and System for the system, that receives as arguments a multiset of natural numbers (the idle processes), a sorted list of numbers (the processes waiting to enter the critical section), a value of sort NatWithEmpty (the process in the critical section), and another multiset of numbers (the processes that have already entered the critical section):

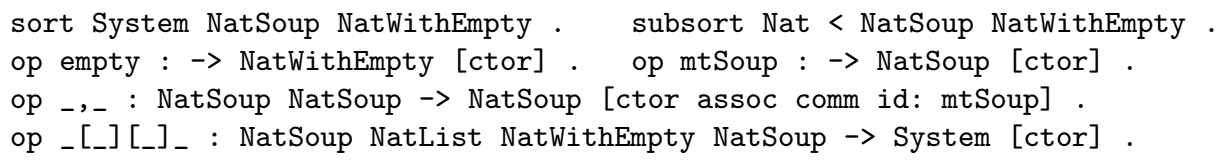

We use the rule ticket to introduce a new process into the list of waiting processes:

$$
\text { rl [ticket] : (N, NS) [NL] [NWE] NS' } \Rightarrow \text { NS [ins-list (NL, N)] [NWE] NS' . }
$$

If at least one process is waiting to enter the critical section and it contains the value empty, then the first process in the list is introduced into the critical section:

$r l[c s-i n]: N S[N$. NL] [empty] NS' $\Rightarrow$ NS [NL] [N] NS' .

The rule cs-out moves the process from the critical section to the finished section:

rl [cs-out] : NS [NL] [N] NS' $\Rightarrow \mathrm{NS}$ [NL] [empty] (N, NS') .

Finally, the rule reset moves the elements in the fourth component of the system to the first one to start the process again:

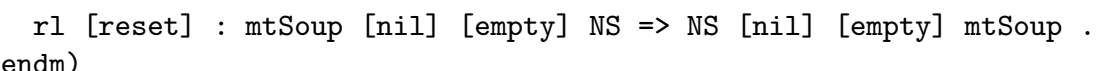

\section{$3.2 \quad$ Narrowing}

Narrowing [3112 23] is a generalization of term rewriting that allows free variables in terms and replaces pattern matching by unification in order to reduce 
these terms. It was first used for solving equational unification problems [29] and then generalized to deal with problems of symbolic reachability. Similarly to rewriting, where at each rewriting step one must choose which subterm of the subject term and which rule of the specification are going to be considered, at each narrowing step one must choose which subterm of the subject term, which rule of the specification, and which instantiation on the variables of the subject term and the rule's lefthand side are going to be considered. The difference between a rewriting step and a narrowing step is that in both cases we use a rewrite rule $l \Rightarrow r$ to rewrite $t$ at a position $p$, but narrowing unifies the lefthand side $l$ and the chosen subject term $t$ before actually performing the rewriting step, while in rewriting this term must be an instance of $l$ (i.e., only matching is required). Using this narrowing approach, we can obtain a substitution that, applied to an initial term that only contains variables (except for the function symbol at the top), generates the most general term that can apply the traversed rules.

We denote by $t \rightsquigarrow^{\sigma} t^{\prime}$, with $\sigma=q_{1} ; \ldots ; q_{n}$ a sequence of labels, the succession of narrowing steps applying (in the given order) the statements $q_{1} ; \ldots ; q_{n}$ that leads from the initial term $t$ (possibly with variables) to the term $t^{\prime}$, and by $\theta_{\sigma}$ the substitution used by this sequence, which results from the composition of the substitutions obtained in each narrowing step. We will overload the notation $t \rightsquigarrow q t^{\prime}$ by using conditions in $q$ to illustrate narrowing steps due to conditions.

In the example above, we could start from the term NS1 [NL] [NWE] NS2, with NS1 and NS2 variables of sort NatSoup, NL a variable of sort NatList, and NWE a variable of sort NatWithEmpty, and apply one step of narrowing to obtain a set of four terms, each of them corresponding to the application of one of the rules for System. For example,

NS1 [NL] [NWE] NS2 $\rightsquigarrow^{\text {ticket }}$ NS3 [ins-list (NL, N1)] [NWE] NS2

where NS1 has been replaced by N1, NS3 (with N1 and NS3 fresh variables of sorts Nat and NatSoup, respectively) and then the rule ticket has been applied.

The latest version of Maude includes an implementation of narrowing for free, $\mathrm{C}, \mathrm{AC}$, or ACU theories in Full Maude [9. More specifically, we are interested in the metaNarrowSearchPath function that, given a term and a bound on the number of narrowing steps, returns all the possible paths starting from this term, the used substitutions, and the applied rules. We use this command to perform a breadth-first search of the state space. Note that the current implementation of narrowing only works for non-conditional rules and specifications without membership axioms; we will show in Section 5 how to check separately the conditions, including membership conditions.

\section{A Module Transformation for Narrowing}

We present in this section a simple module transformation that will be applied to the modules in order to use narrowing with the equational part of Maude. 
This transformation has two objectives: on the one hand it transforms equations into rules (and thus it requires to transform equational conditions into rewrite conditions), which allows us to use narrowing with the equational part of Maude system modules. On the other hand, and since the current implementation of narrowing in Maude does not support memberships, we transform all the terms where membership inferences may be needed into equivalent terms with the variables declared at the kind level, while extra membership conditions stating the correct sort, that will be separately checked with the mechanisms in the next section, are added for each variable whose type has changed. More specifically, the transformation takes an equation of the form

$$
l=r \text { if } \bigwedge_{i=1}^{n} t_{i}=t_{i}^{\prime} \wedge \bigwedge_{j=1}^{m} p_{j}:=u_{j} \wedge \bigwedge_{k=1}^{l} v_{k}: s_{k}
$$

and returns a rule

$$
\begin{aligned}
\operatorname{kind}(l) \Rightarrow \operatorname{kind}(r) \text { if } & m b s(l) \wedge \\
& \bigwedge_{i=1}^{n}\left(\operatorname{kind}\left(t_{i}\right) \Rightarrow w_{i} \wedge \operatorname{kind}\left(t_{i}^{\prime}\right) \Rightarrow w_{i}\right) \wedge \\
& \bigwedge_{j=1}^{m}\left(\operatorname{kind}\left(u_{j}\right) \Rightarrow \operatorname{kind}\left(p_{j}\right) \wedge \operatorname{mbs}\left(p_{j}\right)\right) \wedge \\
& \bigwedge_{k=1}^{l} \operatorname{kind}\left(v_{k}\right): s_{k}
\end{aligned}
$$

where

- The terms $w_{i}$ are fresh variables of the same kind as the corresponding term.

- The function kind replaces the sort of all the variables in the term given as argument by the corresponding kind (we follow here the Maude approach that represents each variable as a pair of an identifier and a type, that can be either a sort or a kind; thus, we can modify these pairs when the second component is a sort by the appropriate kind).

- The function mbs generates a conjunction of conditions stating that the variables, whose type has been changed by its kind, have in fact the sort previously required, that is:

$$
\begin{aligned}
\operatorname{mbs}\left(f\left(t_{1}, \ldots, t_{n}\right)\right) & =m b s\left(t_{1}\right) \wedge \cdots \wedge m b s\left(t_{n}\right) \\
m b s(c) & =\operatorname{nil} \\
m b s(v) & =\operatorname{kind}(v): \operatorname{sort}(v)
\end{aligned}
$$

where $f$ is a function symbol, the $t_{i}$ are terms, $c$ is a constant, $v$ is a variable, and $\operatorname{sort}(v)$ returns the sort of $v$.

We have to transform similarly all the membership axioms and rules in the module in order to apply them. In the membership case we obtain another membership axiom with the lefthand side and the condition transformed as shown above 2 while rules are transformed into rules, being the equational part transformed as in the previous cases while the rewriting conditions remain unchanged.

\footnotetext{
${ }^{2}$ Note that this transformation may generate invalid membership axioms, because they may contain rewrite conditions. However, in practice all the equations and rules in the module are unconditional and the membership axioms have been removed in order to use narrowing; these conditions and membership axioms are kept apart and checked separately by using the techniques described in Section 5
} 
Note that, since Maude equational modules are assumed to be confluent and terminating, the equations may be understood as oriented from left to right, which is what we are explicitly doing when transforming them into rules. Moreover, the kind transformation only postpones (but not prevents from) the checking of the specific sorts of the variables to the condition of the rule. For these reasons, it is straightforward to see that this transformation is correct, even though it can only be executed by using narrowing as explained in the next section.

If we transform the critical section example above, the membership axiom ol2 is modified as follows (assume that the variables are now declared at the kind level):

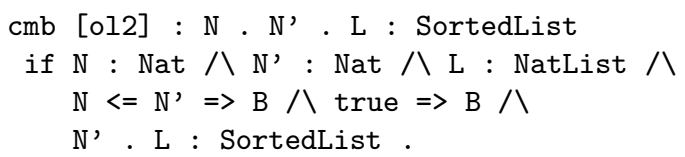

The first three conditions indicate that the variables, that are now declared at the kind level, have in fact the appropriate sort. The next two conditions deal with the first condition of the original axiom, $\mathrm{N}<=\mathrm{N}^{\prime}$, which is an abbreviation for $\mathrm{N}<=\mathrm{N}^{\prime}=$ true; in this case both sides of the equality must be rewritten to the same variable B, defined in the kind of Bool. Finally, the membership condition remains unchanged.

In a similar way, the equation il2 is transformed into the following rule:

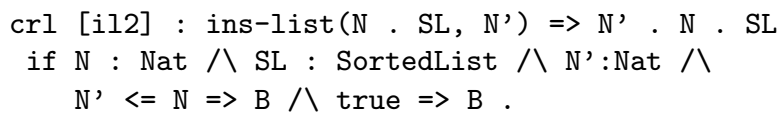

where the first three conditions indicate that the variables have the appropriate sort. The next two conditions deal with the condition of the original axiom, $\mathrm{N}^{\prime}<=\mathrm{N}^{\prime}$, , which is an abbreviation for $\mathrm{N}^{\prime}<=\mathrm{N}^{\prime}=$ true; in this case both sides of the equality must be rewritten to the same variable $\mathrm{B}$, defined in the kind of Bool.

\section{$5 \quad$ Narrowing of Conditional Rules}

We present in this section a methodology to take into account the conditions in the narrowing process because, as explained in the introduction, they are not supported by the current implementation of the Maude system. Note that other systems deal with rewrite conditions (see e.g. [2316]) with a similar approach to ours: they must be solved before applying the body of the rule. The novelty of our technique, beyond describing and implementing this narrowing of conditional rules in Maude, lies on the resolution of membership conditions by means of unification.

Basically, when a rule is applied the conditions must be evaluated separately by using narrowing (remember that equational conditions become rewrite conditions) to find a substitution (that must be the composition of the substitutions 
obtained for each single condition) that fulfills them. If the set of substitutions fulfilling the conditions is nonempty, all of them extend the set of substitutions obtained for the unconditional rule; otherwise, the rule cannot be applied.

However, in addition to rewrite conditions we must also take into account membership conditions. The current implementation of narrowing does not support membership axioms, and thus we must independently check whether a membership condition holds. The first step to achieve it was presented in the previous section: we transform the lefthand of the statements to deal with kinds instead of sorts in order to move the membership information to the conditions. The next step consists of checking the memberships (those introduced by the transformation, as well as those stated by the user); if the sort is defined by using membership axioms (and possibly by operators), then we unify the current term with the lefthand side of each membership axiom inferring this sort or any of its subsorts and then we proceed to prove the conditions in the corresponding axioms as explained before, applying the substitution obtained in the unification (moreover, it also updates the type of the variables, if they are at the kind level, to the required sorts in order to use the operator definitions, see the example below for details). Otherwise (the sort is not defined by using memberships) we update the type of the variables and the rest of the condition is processed.

In our example, we can apply conditional narrowing to ins-list (NL, N1). The narrowing process would start by unifying this term with the lefthand side of $i 12 \sqrt[3]{3}$ whose transformed version was presented at the end of the previous section:

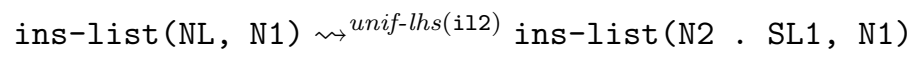

This first step requires the initial list of natural numbers NL to be of the form N2 . SL1, being N2 and SL1 fresh variables at the kind level. Thus, the unification generates the substitution NL $\mapsto N 2$. SL1. However, it must be extended by using the conditions of the applied rule. The first condition, $\mathrm{N}$ : Nat, is a membership condition for a sort that is not defined with membership axioms, and thus it forces the variable $\mathrm{N}^{4}$ to have sort Nat; we change the sort of the variable and proceed with the next condition. The second condition, SL : SortedList, is trickier because this sort is defined by means of membership axioms. We must use a transformed version of the membership axiom ol2 to obtain:

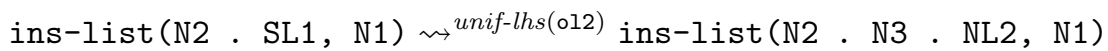

where the unification of the term with the lefthand side of the membership axiom gives the substitution SL1 $\mapsto$ N3 . NL2. Note that the transformation of o12 generates three initial conditions $\left(\mathrm{N}:\right.$ Nat $八 \mathrm{~N}^{\prime}:$ Nat $八 \mathrm{~L}:$ NatList) that

\footnotetext{
${ }^{3}$ Note that other rules, such as il1 or il3, could be also used. In the same way, some other steps in this example could apply different membership axioms and rules.

${ }^{4}$ Note that, after the unification, the rule is being symbolically applied by using the substitution $\mathrm{N} \mapsto \mathrm{N} 2$; SL $\mapsto$ SL1 ; N' $\mapsto \mathrm{N} 1$. In the following, we will not show the substitution required to apply each rule.
} 
just update the sorts of the variables, two rewrite conditions, $\mathrm{N}<=\mathrm{N}$ ' $\Rightarrow \mathrm{B}$ and true $\Rightarrow>B$, which require narrowing again to be solved, and keeps the membership condition unmodified. As we will explain below, since our implementation supports some predefined operators such as $<=$, that returns true when its first argument is 0 , we can use narrowing to solve the rewrite conditions:

ins-list (N2 . N3 . NL2, N1) $\rightsquigarrow^{N^{<}<N^{\prime} \Rightarrow B}$ ins-list (0.N3 . NL2, N1)

and the current substitution is extended with $\mathrm{N} 2 \mapsto 0 ; \mathrm{B} \mapsto$ true. With this substitution the next condition of ol2 ( $\mathrm{B} \Rightarrow$ true) trivially holds and only the membership condition, $N^{\prime}$. L : SortedList, remains. It can be satisfied by using the membership axiom ol1, which extends the substitution with NL2 $\mapsto$ nil. Summarizing the narrowing process thus far, starting from the term ins-list(NL, N1) and applying the rule il2 and its two first conditions (which includes applying the membership axiom ol2 and all its conditions, the rule for $<=$, and the membership axiom ol1), we have reached ins-list ( 0 . N3 . nil, N1) with the substitution NL $\mapsto 0$. N3 . nil. We proceed now with the third condition of $i 12, N^{\prime}:$ Nat, that simply updates the sort of $\mathrm{N} 1$. The next condition, $\mathrm{N}^{\prime}<=\mathrm{N} \Rightarrow \mathrm{B}$, is solved as explained above by using the substitution $\mathrm{N} 1 \mapsto 0 ; \mathrm{B} \mapsto$ true:

$$
\text { ins-list (0.N3. nil, N1) } \rightsquigarrow^{N 1<=0 \Rightarrow B} \text { ins-list }(0 \text {. N3 . nil, 0) }
$$

Finally, the last condition for il2 holds because true is rewritten to itself, and the rule is applied by using the obtained substitution in the righthand side, obtaining the following complete narrowing step with the substitution NL $\mapsto$ 0 . N3 . nil; N1 $\mapsto 0$ :

$$
\text { ins-list (NL, N1) } \rightsquigarrow^{i 12} 0 \cdot 0 \text {. N3 . nil }
$$

\subsection{A Brief Note on Predefined Functions}

As we have shown in the example above, we use some predefined functions on the narrowing process. We basically add some rules to deal with the most used functions for boolean values and natural numbers. For example, we add the rules

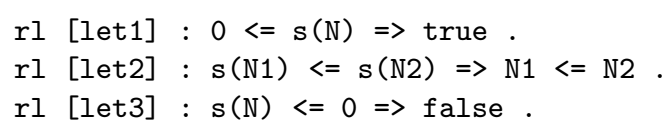

to deal with the _ $<=$ _ function. In this way we introduce rules are easily managed by the narrowing mechanisms and greatly increase the number of Maude specifications that can be tested with the tool.

\section{Using Narrowing to Generate Test Cases}

Different testing techniques can be used to test Maude specifications, and for each of these techniques a different narrowing strategy will be used. We show 
in this section how to compute a coverage, how to check whether a specification fulfills an invariant, and how to examine if, given a correct specification, another module performs the required actions, which is called conformance testing.

\subsection{Coverage Criteria}

Code coverage techniques 1925 consist of selecting a set of test cases that, when executed, apply all the statements required by the coverage criterion. In our case we use global branch coverage [13, a strategy that tries to find test cases that use all the statements potentially used by the function under test (which, of course, includes the functions and membership axioms in the conditions) and has been already described for functional modules in [27, and system coverage, an adaptation of modified condition decision coverage 19 that tries to obtain information by making the conditions to fail.

Narrowing can be naturally used to compute global branch coverage by starting with a term with variables and performing a breadth-first search, where after each narrowing step, that computes the set of reachable terms by applying one rule, we check that the conditions of each rule are fulfilled by using the mechanism presented in the previous section, thus removing some of the obtained terms and extending the substitutions when required (e.g., in the example of the previous section, the substitution was extended to $\mathrm{NL} \mapsto 0$. N3 . nil ; N1 $\mapsto 0$ ). This search finishes when all the statements required by the coverage have been used, a bound in the number of steps has been reached, or all the possible states have been reached (this last point is checked by trying to unify the terms obtained in each step with any of the previous terms; that is, we build a graph instead of a tree). Moreover, our system provides two different options to select the set of test cases: the smallest one, composed of the minimum number of terms whose execution leads to the execution of all the statements in the coverage and thus may contain complex test cases; and the simplest one, in the sense that it may present more but simpler test cases. The user can switch between these two modes to decide which one is more appropriate for each specification.

More formally, we look for a set of sequences $\sigma_{i}$ and terms $t_{i}, 0 \leq i \leq l$, such that, given the set of labels $Q$ defining the coverage and a term $f\left(v_{1}, \ldots, v_{n}\right)$, with $f$ the function under test and $v_{i}$ variables of the appropriate sorts, $\forall q \in Q \exists_{i=0}^{l} \cdot f\left(v_{1}, \ldots, v_{n}\right) \rightsquigarrow^{\sigma_{i}} t_{i} \wedge q \in \sigma_{i}$. The test cases will be $\bigcup_{i=0}^{l} \theta_{\sigma_{i}}\left(f\left(v_{1}, \ldots, v_{n}\right)\right)$. Since there are several different possibilities to select the $\sigma_{i}$, the different strategies to display the set of test cases will choose between a small number of large sequences, that will generate less test cases applying more rules, and a big number of short sequences, that will generate simpler test cases. Note that the extension to testing of system modules is straightforward by starting from $c\left(v_{1}, \ldots, v_{n}\right)$, with $c$ any constructor for the sort being tested.

In our lists example, we may be interested in testing the function ins-sort using the global branch coverage criterion. This function is defined by two equations (is1 and is2); one of these equations uses the function ins-list, and thus its three equations (il1, il2, and il3) must also be added to the needed coverage; finally, this function uses the functions _ $<$, and ${ }_{-}<=_{-}$, imported from 


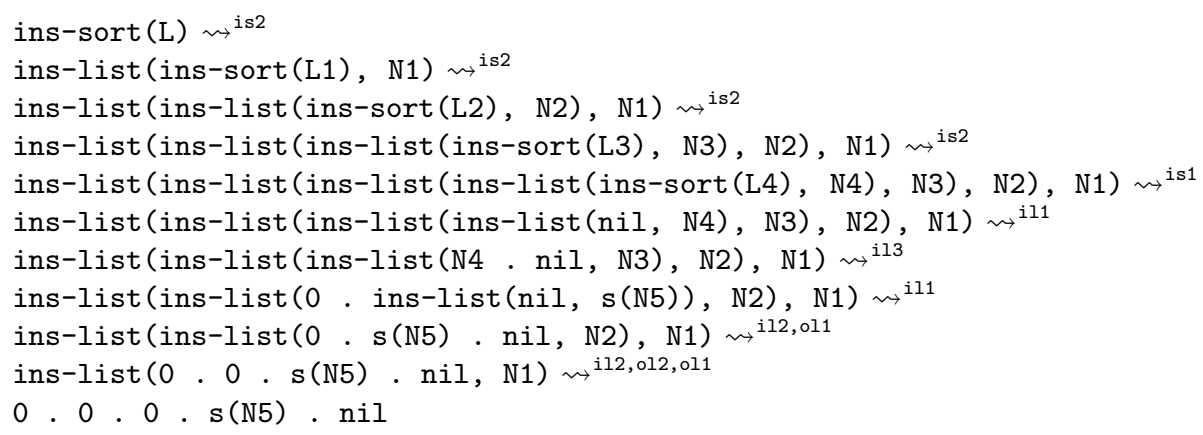

Fig. 1. Narrowing path for global branch coverage

NAT and a variable of sort SortedList, which is defined with two membership axioms (ol1 and ol2). All these statements must be executed at least once by the test cases to fulfill global branch coverage. We can use our tool to automatically generate the test cases, following the default strategy that selects the smaller set of test cases:

Maude> (test ins-sort.)

1. ins-sort (1.0.0.0. nil) has been reduced to 0 . 0 . 0 . 1 . nil All the statements were covered.

Note that the tool shows the initial term, the result of reducing it in the module, and whether some reachable statements could not be used. The term shown by the tool may be obtained as shown in Figure 1. where s stands for the successor function over natural number (note that this is one branch of a search tree of depth 10).

Since all the possible instantiations of this term generate test cases traversing all the required statements, the tool generates the simplest one by replacing the variables with constants of the given sort (or the simplest built term if the sort does not have constants). If we find that any reduction is wrong, we could debug it with:

Maude> (invoke debugger with user test case 1. )

Declarative debugging of wrong answers started.

This command starts the declarative debugging process [28] that, by asking questions to the user about the computations that took place will find the specific statement that generated the wrong behavior. This command is available for all the testing options.

Note that the extension to testing of system modules is straightforward; in this case we want to test the transitions of the terms with a given sort instead of a specific function, and thus the narrowing process starts with a term with variables of the given sort, and tries to apply all the reachable rules, equations, and memberships, proceeding in the same way as the testing for functional modules. That is, we start the narrowing process from $c\left(v_{1}, \ldots, v_{n}\right)$, with $c$ any constructor 
for the sort and $v_{i}$ variables of the appropriate sorts, and continue as indicated above.

Moreover, we propose another coverage criterion, related to modified condition decision coverage $(\mathrm{MCDC})$ 1910. Basically, MCDC requires that all the conditions in a program are evaluated with the given set of test cases to both true and false. In a non-deterministic framework as the one of system modules it is important to know, as explained in [28, the applied statements that make the program reach certain states, the positive information, but also the statements that were not applied and thus prevented the program from reaching some other values, the negative information. While we obtain the positive information with the global branch coverage shown above, it does not provide any of the negative information. For this reason, we have implemented the so-called system coverage criterion, which requires a set of test cases to apply all the rules in the transformed module (which corresponds to global branch coverage) but also to fail for at least one condition for each rule in the original module.

\subsection{Checking Invariants}

Checking of invariants has already been studied for Maude specifications in 8 , Chapter 12]. It takes advantage of the command search, that performs a breadthfirst search from an initial term, given a bound in the number of steps and a condition to be fulfilled; by searching for the negation of the invariant we can check that no illegal states are reached. We apply a similar idea in our testing framework by using symbolic search; this search will traverse all the possible states and, each time a rule is applied, it tries to find a path to fulfill the negation of the invariant. If such a path is found, then the specification does not fulfill the invariant. Note that the invariant is usually specified by using equations, and thus it is important to use equations in the narrowing process, since it allows the tool to fix the values of the initial state required to fulfill the condition.

More formally, we consider a new rule inv $($ pat $) \Rightarrow$ pat if Cond, where inv is a new operator defined over the sort of states, pat is the pattern given for the invariant, and Cond a condition (we assume the invariant is composed of a pattern and a condition, see below for details). Thus, for every narrowing sequence $t \rightsquigarrow q_{1} t_{1} \rightsquigarrow^{q_{2}} \ldots \rightsquigarrow q_{n} t_{n}$, the invariant is fulfilled if, for every $t_{i}$ obtained by using a narrowing step with the rule $q_{i}$ we cannot find a term $t^{\prime}$ such that $t_{i} \rightsquigarrow^{i n v} t^{\prime}$ (we look for the negation of the invariant). If such a term exists, then the term $\theta_{i n v}\left(\theta_{q_{1} ; \ldots ; q_{i}}(t)\right)$ can be used as initial term for debugging; otherwise, the invariant holds.

The transformation presented in Section 4 allows us to check invariants in both functional and system modules. We could e.g. set an invariant on our critical section example stating some correct property over lists or systems, but it is worth examining how an initial term proving the specification wrong is obtained. In our critical section example we can specify a function empty?, that checks whether a NatSoup is empty, defined as follows: 


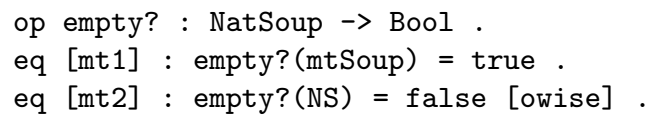

and then search for a system that never has its first argument empty (remember that we look for the negation of the invariant) with:

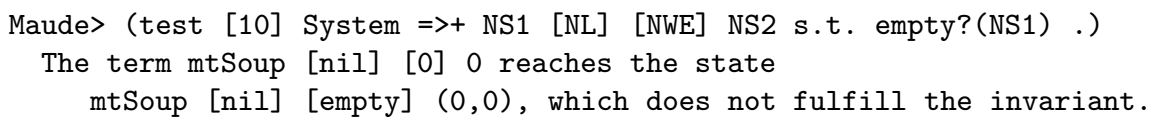

This command looks for terms of sort System that, in at least one step (indicated by the search arrow $=>+$; the tool also provides searches in zero or more steps with $=>*$ and searches for final forms with $=>$ !) and at most 10 , match the pattern and fulfill the condition (the negation of the invariant). In this case, the tool has found (as expected) an initial state that, after applying one rule (in this specific case it is cs-out although it would be possible to apply other rules), reaches a state that does not fulfill the invariant. In this case the narrowing process has fixed the value of the first NatSoup to mtSoup to fulfill the condition and has forced an element to be in the critical section to apply the rule, while the nil list and the singleton soup are just possible instances of the variables left by the narrowing step NS1 [NL] [NWE] NS2 $\rightsquigarrow^{\text {cs-out }} \mathrm{NS} 1$ [NL] [empty] (N1, NS2) and then checking the property by instantiating NS1 with mtSoup when applying the equations for empty?.

\subsection{Conformance Testing}

Conformance testing [32 145] involves testing a system with respect to its specification. The goal of this approach is to check that the system presents the same behavior as the specification, that has already been tested. To check whether an implementation conforms to a specification we must formalize the conformance notion by means of an implementation relation that relates the two systems. In our case, and taking into account that a rewrite system can be understood as a labeled transition system, where terms stand for states and rewrites for transitions, we apply to Maude specifications the conformance testing strategies for such systems [32]. In particular, we use the relation conf [4], that requires the implementation to perform the same actions as the specification, although it allows the implementation to execute some other actions not included in the specification, that is, conf requires that an implementation does what it should do, not that it does not do what it is not allowed to do.

In our framework we consider that only the rules in the original specification must be executed in the implementation, and thus narrowing steps using equations are considered auxiliary and it is not required to reproduce them in the implementation. In this way, we compute all the possible paths by using narrowing in the specification and then that all these paths are also possible in the implementation. More formally, if we denote by $\left.\sigma\right|_{R}$ the restriction of $\sigma$ to the rules in $R$, that is, remove from $\sigma$ all those statements that are not in the set, then we require that for every narrowing sequence $t \rightsquigarrow \sigma_{s} t_{s}$ in the specification 
there exists a narrowing sequence $t \rightsquigarrow^{\sigma_{i}} t_{i}$ in the implementation such that $\left.\sigma_{s}\right|_{R}$ is a prefix of $\left.\sigma_{i}\right|_{R}$. Note that, although the reached states may be different in the specification and the implementation (only the applied rules matter), we consider that both the correct specification and the system being tested share the same signature for the initial terms and the same rule labels; this can be achieved by means of a renaming.

For the sake of example, we could create a new module RED-CS that has the same rules as CS except for the rule cs-out. We can state CS as the specification with:

Maude> (correct test module CS .)

CS selected as correct module for testing.

Now, we can check the behavior of RED-CS with respect to this module with:

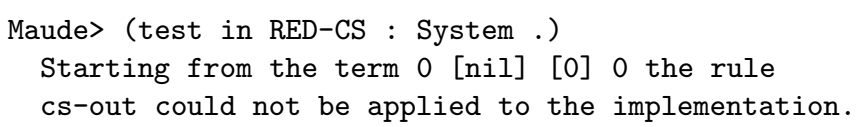

That is, the tool shows the simplest term (in fact, only the 0 in the critical section is instantiated during the process) that is required to find the disconformity between the specification and the implementation due to the cs-out rule.

\section{Trusting}

Our tool provides some trusting techniques to enhance its performance. Basically, it only takes into account labeled statements when computing coverages and checking the implementation relation. Moreover, the user can also select a subset of these statements by using the different commands available in the tool (trusting of all the statements of a given module, trusting of a complete kind of statements - e.g. all the equations, memberships, or rules - and trusting of single statements). Using these commands we can use different trusting strategies: assuming that our specifications are structured, we can test first easier specifications, and then trust them when testing larger specifications including them; and we can trust all the equations (except for the ones defining the property when checking invariants) and memberships when testing system modules. Of course, trusting mechanisms are correct assuming the user points out as trusted only rules that are not relevant for the testing process.

Trusting works in a different way depending on the testing strategy: if we are computing a coverage then the trusted statements are removed from the needed coverage, and thus we may reduce both the number and the complexity of the test cases. When using conformance testing, the trusted statements are related to the specification and indicate that the behavior specified by the rule is not required to be performed in the system being tested (e.g. because it is an auxiliary rule). That is, the sequences of statements $\sigma$ required for coverage are not required to contain the trusted statements 5 while the restriction to rules

\footnotetext{
${ }^{5}$ Note that using trusting when using system coverage will remove the statements from both the positive and negative information.
} 


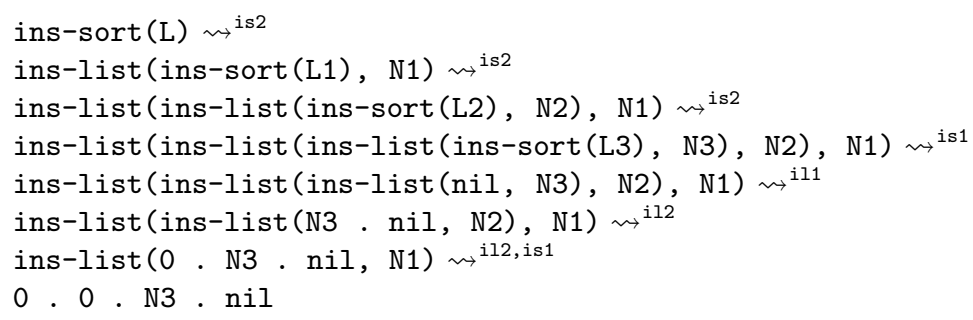

Fig. 2. Narrowing path for global branch coverage with trusting

in the specification given in conformance testing is now applied to non-trusted rules in the specification.

For example, we can trust the statements il3 and ol2 (which required the longest computations in Figure 1) if we are sure of its correctness to improve the performance of the computation of the global branch coverage in Section 6.1 by using the commands:

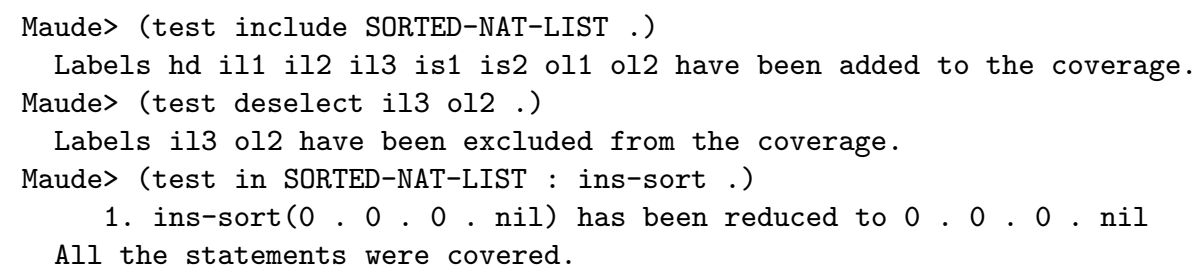

Obtaining in this case a simpler test case that covers all the statements. It is interesting to see that trusting a rule when using conformance provides more flexibility, because it allows to perform some analyses by removing auxiliary rules that are not supposed to be applied in the final implementation. However, if the user trusts a statement that should not be trusted he may obtain an incorrect answer, hence the assumption presented above about the correctness of trusting, that may produce incorrect results. Similarly, we can trust the rule cs-out when using conformance testing and check that in this case the specification and the implementation perform the same actions:

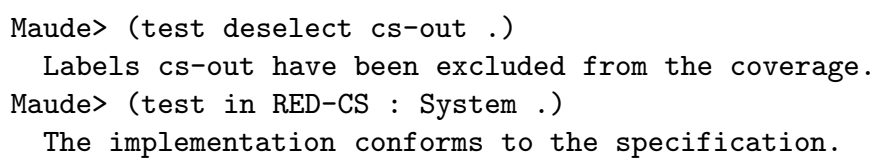

The improvement in the performance when using trusting is highly dependent on the selected set of statements: while in some cases trusting may reduce the number of steps more than a 50\%, in other cases they are not reduced at all. For example, the global branch coverage obtained in Section 6.1 was highly reduced by trusting the statements shown above, reducing the depth of the search tree from 10 to 7 , as illustrated in Figure 26 However, selecting other statements

\footnotetext{
${ }^{6}$ Remember that this is one branch of the search tree, that is, trusting has reduced the depth of the search tree from 10 to 7 , which results in a huge improvement of the performance.
} 
such as is1 or is2, that must be always executed in order to reach a state where other statements can be used, would not reduce the size of the search at all. All the examples in this paper, and much more information is available at http://maude.sip.ucm.es/testing/.

\section{Concluding Remarks and Ongoing Work}

We have presented in this paper how to use narrowing to generate test cases for Maude specifications. To achieve this we use a module transformation that allows us to use the equational part of Maude modules in the narrowing process and a method to check whether the conditions of the applied statements are fulfilled, including those conditions that require membership axioms. Using these techniques we have implemented a tool that is able to compute a set of test cases fulfilling two different coverage criteria, to check whether an invariant is fulfilled by the specification, and to examine whether an implementation of the system fulfills the behavior indicated by its specification. Moreover, two different sets of test cases can be computed: a smaller set that contains more complex terms or a larger set that contains less complex terms; the user is in charge of selecting the most appropriate depending on the complexity of the specification and his knowledge of it. Trusting mechanisms are also provided to improve the performance of both coverage criteria and conformance testing. Finally, some predefined modules can be also used to generate the test cases. We are currently working on a comparison between our current approach using narrowing and (i) the previous one using ground terms, and (ii) similar approaches in other languages, either using narrowing, like Lazy Smallcheck, or random testing, like QuickCheck.

As future work, we plan to extend the tool by introducing symbolic model checking [1], that would allow the user to check linear temporal logic formulas over the specification starting from a term with variables, thus proving the formula on, potentially, all the possible inputs of the system. Moreover, we are studying new coverage criteria and implementation relations to allow the user to choose the most appropriate technique for each application. Finally, we also intend to develop a distributed implementation of the tool to deal with narrowing; in this way, we can start the symbolic search of the system in one Maude instance and then send the different paths to different Maude processes, that must share some information (the coverage and the reached states) to finish the search as soon as possible.

Acknowledgements. I thank Santiago Escobar for his kind help with narrowing.

\section{References}

1. Beizer, B.: Software testing techniques. Dreamtech (2002)

2. Borba, P., Cavalcanti, A., Sampaio, A., Woodcook, J. (eds.): PSSE 2007. LNCS, vol. 6153. Springer, Heidelberg (2010) 
3. Bouhoula, A., Jouannaud, J.-P., Meseguer, J.: Specification and proof in membership equational logic. Theoretical Computer Science 236, 35-132 (2000)

4. Brinksma, E., Scollo, G., Steenbergen, C.: LOTOS specifications, their implementations and their tests. In: Protocol Specification, Testing, and Verification VI, pp. 349-360 (1987)

5. Cartaxo, E.G., Neto, F.G.O., Machado, P.D.L.: Test case generation by means of UML sequence diagrams and labeled transition systems. In: Proceedings of the IEEE International Conference on Systems, Man and Cybernetics, SMC 2007, pp. 1292-1297. IEEE (2007)

6. Christiansen, J., Fischer, S.: EasyCheck - Test Data for Free. In: Garrigue, J., Hermenegildo, M.V. (eds.) FLOPS 2008. LNCS, vol. 4989, pp. 322-336. Springer, Heidelberg (2008)

7. Claessen, K., Hughes, J.: Quickcheck: A lightweight tool for random testing of Haskell programs. ACM SIGPLAN Notices, 268-279 (2000)

8. Clavel, M., Durán, F., Eker, S., Lincoln, P., Martí-Oliet, N., Meseguer, J., Talcott, C.: All About Maude - A High-Performance Logical Framework. LNCS, vol. 4350. Springer, Heidelberg (2007)

9. Clavel, M., Durán, F., Eker, S., Lincoln, P., Martí-Oliet, N., Meseguer, J., Talcott, C.: Maude Manual (Version 2.6) (January 2011), http://maude.cs.uiuc.edu/maude2-manual

10. Dupuy, A., Leveson, N.: An empirical evaluation of the MC/DC coverage criterion on the HETE-2 satellite software. In: Proceedings of the 19th Digital Avionics Systems Conference, DASC 2000, vol. 1, pp. 1B6.1-1B6.7 (2000)

11. Escobar, S., Meseguer, J.: Symbolic Model Checking of Infinite-State Systems Using Narrowing. In: Baader, F. (ed.) RTA 2007. LNCS, vol. 4533, pp. 153-168. Springer, Heidelberg (2007)

12. Fay, M.: First-order unification in an equational theory. In: Joyner, W.H. (ed.) Proceedings of the 4th Workshop on Automated Deduction, pp. 161-167. Academic Press (1979)

13. Fischer, S., Kuchen, H.: Systematic generation of glass-box test cases for functional logic programs. In: Proceedings of the 9th ACM SIGPLAN International Conference on Principles and Practice of Declarative Programming, PPDP 2007, pp. 63-74. ACM Press (2007)

14. Gaudel, M.-C.: Software Testing Based on Formal Specification. In: Borba, P., Cavalcanti, A., Sampaio, A., Woodcook, J. (eds.) PSSE 2007. LNCS, vol. 6153, pp. 215-242. Springer, Heidelberg (2010)

15. Gomez-Zamalloa, M., Albert, E., Puebla, G.: Test case generation for objectoriented imperative languages in CLP. Theory and Practice of Logic Programming 10, 659-674 (2010)

16. Hussmann, H.: Unification in Conditional Equational Theories. In: Caviness, B.F. (ed.) EUROCAL 1985. LNCS, vol. 204, pp. 543-553. Springer, Heidelberg (1985)

17. Hierons, R.M., Bogdanov, K., Bowen, J.P., Rance Cleaveland, J.D., Dick, J., Gheorghe, M., Harman, M., Kapoor, K., Krause, P., Lüttgen, G., Simons, A.J.H., Vilkomir, S., Woodward, M.R., Zedan, H.: Using formal specifications to support testing. ACM Computing Surveys 41(2), 1-76 (2009)

18. Jacquemard, F., Rusinowitch, M., Vigneron, L.: Compiling and Verifying Security Protocols. In: Parigot, M., Voronkov, A. (eds.) LPAR 2000. LNCS (LNAI), vol. 1955, pp. 131-160. Springer, Heidelberg (2000) 
19. Jasper, R., Brennan, M., Williamson, K., Currier, B., Zimmerman, D.: Test data generation and feasible path analysis. In: Proceedings of the 1994 ACM SIGSOFT International Symposium on Software Testing and Analysis, ISSTA 1994, pp. 95-107. ACM (1994)

20. King, J.C.: Symbolic execution and program testing. Communications of the ACM 19, 385-394 (1976)

21. Meadows, C.: Applying formal methods to the analysis of a key management protocol. Journal of Computer Security 1 (1992)

22. Meseguer, J.: Conditional rewriting logic as a unified model of concurrency. Theoretical Computer Science 96(1), 73-155 (1992)

23. Middeldorp, A., Hamoen, E.: Counterexamples to Completeness Results for Basic Narrowing. In: Kirchner, H., Levi, G. (eds.) ALP 1992. LNCS, vol. 632, pp. 244-258. Springer, Heidelberg (1992)

24. Müller, R.A., Lembeck, C., Kuchen, H.: A symbolic Java virtual machine for test case generation. In: IASTED Conf. on Software Engineering, pp. 365-371 (2004)

25. Ntafos, S.C.: A comparison of some structural testing strategies. IEEE Transactions on Software Engineering 14, 868-874 (1988)

26. Pacheco, C.: Directed Random Testing. PhD thesis, Massachusetts Institute of Technology (June 2009)

27. Riesco, A.: Test-Case Generation for Maude Functional Modules. In: Mossakowski, T., Kreowski, H.-J. (eds.) WADT 2010. LNCS, vol. 7137, pp. 287-301. Springer, Heidelberg (2012)

28. Riesco, A., Verdejo, A., Martí-Oliet, N., Caballero, R.: Declarative debugging of rewriting logic specifications. Journal of Logic and Algebraic Programming (2011) (to appear)

29. Robinson, J.A.: A machine-oriented logic based on the resolution principle. Journal of the ACM 12(1), 23-41 (1965)

30. Runciman, C., Naylor, M., Lindblad, F.: Smallcheck and Lazy Smallcheck: automatic exhaustive testing for small values. In: Gill, A. (ed.) Proceedings of the 1st ACM SIGPLAN Symposium on Haskell, Haskell 2008, Victoria, BC, Canada, September 25, pp. 37-48. ACM (2008)

31. Slagle, J.R.: Automated theorem-proving for theories with simplifiers, commutativity and associativity. Journal of the ACM 21(4), 622-642 (1974)

32. Tretmans, J.: Model Based Testing with Labelled Transition Systems. In: Hierons, R.M., Bowen, J.P., Harman, M. (eds.) Formal Methods and Testing. LNCS, vol. 4949, pp. 1-38. Springer, Heidelberg (2008) 